\title{
A Case of Chronic Intestinal Pseudo-obstruction with Mitochondrial Diseases
}

\author{
Takanobu Jinnouchi ${ }^{1}$, Yoshitaka Sakurai ${ }^{1}$, Kengo Miyoshi ${ }^{1}$, Chie Koizumi ${ }^{1}$, Hironori Waki ${ }^{1}$, \\ Naoto Kubota ${ }^{1,2}$ and Toshimasa Yamauchi ${ }^{1}$
}

\begin{abstract}
:
Chronic intestinal pseudo-obstruction (CIPO) is a rare disorder of intestinal dysmotility characterized by chronic symptoms, including vomiting and abdominal pain, associated with bowel obstruction without any mechanical obstructive causes. We herein report a case of mitochondrial diseases with recurrent duodenal obstruction that was initially diagnosed as superior mesenteric artery syndrome (SMAS) for a few years but was later diagnosed as CIPO. Since CIPO is known to be associated with mitochondrial diseases, it should be considered in the differential diagnosis of patients with mitochondrial diseases presenting with recurrent intestinal obstruction.
\end{abstract}

Key words: mitochondrial diseases, superior mesenteric artery syndrome, chronic intestinal pseudoobstruction

(Intern Med Advance Publication)

(DOI: 10.2169/internalmedicine.7714-21)

\section{Introduction}

Mitochondrial diseases are genetic disorders caused by defects in the mitochondrial metabolism, including the Krebs cycle, $\beta$-oxidation, and lipid and cholesterol synthesis. Mitochondrial diseases arise as a result of mutations in the mitochondrial DNA or nuclear DNA (1) and affect many organs, including the nervous, cardiac, respiratory, endocrine, and gastrointestinal systems. Furthermore, there are diverse clinical phenotypes, including maternally inherited diabetes and deafness (MIDD); mitochondrial myopathy, encephalopathy, lactic acidosis, and stroke-like episodes (MELAS); and others (2). MIDD is characterized by young-onset nonobese diabetes mellitus with impaired insulin secretion, accompanied by neurosensory hearing loss and a maternal family history of diabetes mellitus (3). MELAS is also a multi-organ disease with broad manifestations including stroke-like episodes, dementia, epilepsy, lactic acidemia, and myopathy (4). MELAS and MIDD are known to be associated with the mitochondrial DNA $3243 \mathrm{~A}>\mathrm{G}$ mutation (5-7).

Chronic intestinal pseudo-obstruction (CIPO) is a syn- drome of chronic intestinal obstruction that occurs in the absence of any significant organic lesion obstructing the intestinal lumen, associated with dysfunction of the extrinsic nerves or smooth muscles of the gastrointestinal tract (8). According to a previous survey in Japan, the estimated prevalence of CIPO is 1.00 and 0.80 cases per 100000 men and women, and the estimated incidence is 0.21 and 0.24 cases per 100000 men and women (9). CIPO is classified as primary or secondary, and among the causes of secondary CIPO, mitochondrial diseases are reported to be common $(10,11)$.

Superior mesenteric artery syndrome (SMAS) is also a rare disorder, characterized by gastrointestinal symptoms caused by vascular compression of the horizontal part of duodenum in the angle between the aorta and the superior mesenteric artery. SMAS is usually associated with a reduction in the mesenteric and retroperitoneal fat mass, along with several predisposing conditions causing emaciation (12).

We herein report the case of a patient with recurrent duodenal obstruction who was diagnosed with secondary CIPO associated with mitochondrial diseases that was initially di-

${ }^{1}$ Department of Diabetes and Metabolic Diseases, Graduate School of Medicine, The University of Tokyo, Japan and ${ }^{2}$ Department of Clinical Nutrition Therapy, The University of Tokyo, Japan

Received: April 6, 2021; Accepted: May 24, 2021; Advance Publication by J-STAGE: August 13, 2021

Correspondence to Dr. Toshimasa Yamauchi, tyamau-tky@umin.net 


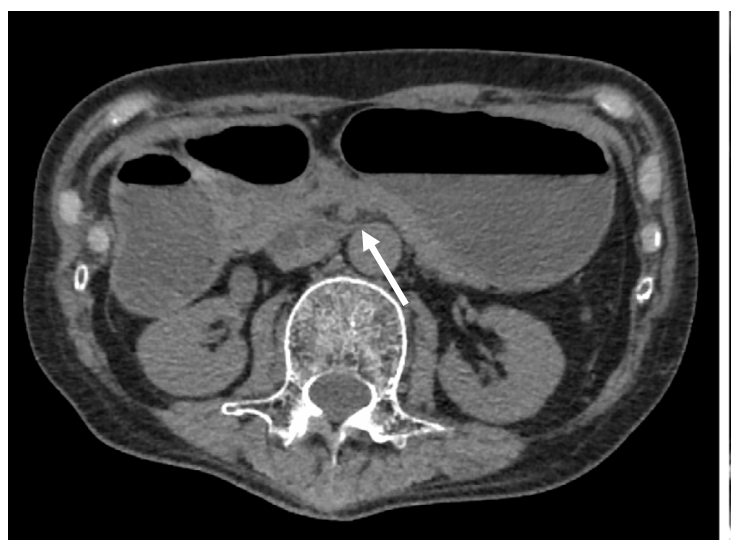

(A)

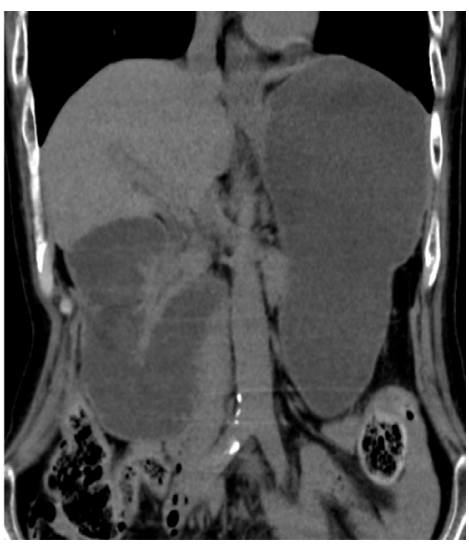

(B)

Figure 1. Computed tomography findings of the abdomen showing the horizontal part of the duodenum compressed between the aorta and superior mesenteric artery (arrow) and dilatation of the stomach and proximal duodenum, with air-fluid levels (A, B).

agnosed as a case of SMAS.

\section{Case Report}

A 62-year-old Japanese man was referred to our hospital with a history of recurrent episodes (every few months to years) of vomiting. At 27 years old, the patient had been diagnosed with diabetes mellitus and was treated with appropriate dietary advice and an oral hypoglycemic agent. He was very lean, with a body mass index (BMI) of around 13 $\mathrm{kg} / \mathrm{m}^{2}$. His HbA1c level had remained at $6 \%-7 \%$ until he was in his $40 \mathrm{~s}$, when it gradually worsened to $8 \%-9 \%$. In addition, the patient also developed gradually worsening hearing loss.

At 51 years old, he had been admitted to our hospital with complaints of poor appetite and worsening glycemic control. At that point, he had been diagnosed with MIDD based on the typical clinical findings, including sensory deafness, decreased insulin secretion, elevated blood lactate/ pyruvate ratio, a maternal family history of diabetes mellitus, and the detection of the mitochondrial DNA 3,243 A>G mutation. After the initiation of treatment with a multiple daily insulin injection regimen, his $\mathrm{HbA1c}$ levels fell again to $6 \%-7 \%$.

At 58 years old, he presented with transient left hemiplegia and was diagnosed with MELAS. At 59 years old, he was hospitalized with repeated episodes of vomiting. Abdominal computed tomography (CT) showed obstruction of the horizontal part of the duodenum, which was compressed between the aorta and the superior mesenteric artery; this led to suspicion of SMAS as the cause of vomiting. His symptoms improved with conservative management.

Subsequently, the patient was readmitted to our hospital with heart failure. Since a myocardial biopsy and electronmicroscopic examination revealed degenerated mitochondria in a variety of sizes and morphologies, the patient was diagnosed with mitochondrial cardiomyopathy. At 62 years old, he presented with vomiting and evidence of duodenal ob- struction, which improved quickly with the insertion of a nasogastric tube. However, nine months later, the patient was admitted to our hospital again with a history of recurrent vomiting. Abdominal CT showed dilatation of the stomach and proximal duodenum and compression of the horizontal part of the duodenum between the aorta and the superior mesenteric artery. The aorta-superior mesenteric artery distance was $4.3 \mathrm{~mm}$ (Fig. 1A and B).

A physical examination on admission revealed that the patient's height was $162.0 \mathrm{~cm}$, and his body weight was $36.2 \mathrm{~kg}$ (BMI: $13.8 \mathrm{~kg} / \mathrm{m}^{2}$ ). There were no significant changes in the laboratory test results (Table). Initially, the patient was diagnosed with recurrent SMAS, based on the CT findings. While gastric fluoroscopy demonstrated decreased peristalsis of the stomach to the duodenum, there was no obstruction at the horizontal part of the duodenum, suggesting that there was no vascular compression of the horizontal part of the duodenum in the angle between the aorta and the superior mesenteric artery. In addition, intestinal peristalsis was not impaired distal to the duodenojejunal flexure (Fig. 2). Thus, secondary CIPO caused by mitochondrial diseases was considered as the underlying etiology for the dilated stomach and proximal duodenum caused by decreased peristalsis at these sites.

While the patient exhibited a blunted Achilles tendon reflex, there were no findings characteristic of diabetic neuropathy, such as a decreased vibration sensation or sensory impairment. Furthermore, the coefficient of variation of the $\mathrm{R}-\mathrm{R}$ interval on and electrocardiogram $\left(\mathrm{CV}_{\mathrm{R}-\mathrm{R}}\right)$ was $2.61 \%$ (normal range, $>1.25 \%$ ), suggesting that there was no severe diabetic autonomic neuropathy. Improvement in the gastrointestinal symptoms was observed after a few days of abstention from oral intake and peripheral parenteral nutrition, without insertion of a nasogastric tube. The patient was discharged from the hospital on Day 21 with pharmacologic treatment, such as mosapride citrate (serotoninergic agents), Daikenchuto (herbal medicine), and magnesium oxide. The clinical course of this patient is shown in Fig. 3. 
Table. Laboratory Tests on Admission.

\begin{tabular}{|c|c|c|c|c|c|c|}
\hline \multicolumn{2}{|c|}{ Complete blood count } & T-Bil & $0.4 \mathrm{mg} / \mathrm{dL}$ & \multirow{2}{*}{\multicolumn{2}{|c|}{$\begin{array}{l}\text { Urinalysis } \\
\text { Protein }\end{array}$}} & $(-)$ \\
\hline WBC & $10,900 / \mu \mathrm{L}$ & BUN & $34.1 \mathrm{mg} / \mathrm{dL}$ & & & $(-)$ \\
\hline $\mathrm{RBC}$ & $433 \times 10^{4} / \mu \mathrm{L}$ & Cre & $0.92 \mathrm{mg} / \mathrm{dL}$ & \multicolumn{2}{|l|}{ Glucose } & $(-)$ \\
\hline $\mathrm{Hb}$ & $13.6 \mathrm{~g} / \mathrm{dL}$ & eGFR & $65.0 \mathrm{~mL} / \mathrm{min} / 1.73 \mathrm{~m}^{2}$ & \multicolumn{2}{|l|}{ Ketone } & $(-)$ \\
\hline \multirow[t]{2}{*}{ PLT } & $27.3 \times 10^{4} / \mu \mathrm{L}$ & UA & $8.0 \mathrm{mg} / \mathrm{dL}$ & \multicolumn{2}{|c|}{ Occult Blood } & $5 \mathrm{mg} / \mathrm{g} \cdot \mathrm{Cre}$ \\
\hline & & CRP & $0.20 \mathrm{mg} / \mathrm{dL}$ & \multicolumn{2}{|c|}{ Microalbumin } & \\
\hline \multicolumn{2}{|c|}{ Blood Chemistry } & $\mathrm{Na}$ & $143 \mathrm{mEq} / \mathrm{L}$ & & & \\
\hline TP & $7.7 \mathrm{~g} / \mathrm{dL}$ & $\mathrm{K}$ & $4.7 \mathrm{mEq} / \mathrm{L}$ & \multicolumn{2}{|c|}{ Meal tolerance test } & \\
\hline Alb & $3.8 \mathrm{~g} / \mathrm{dL}$ & $\mathrm{Cl}$ & $93 \mathrm{mEq} / \mathrm{L}$ & Glucose & $0 \mathrm{~min}$ & $90 \mathrm{mg} / \mathrm{dL}$ \\
\hline LDH & $310 \mathrm{U} / \mathrm{L}$ & Glucose & $250 \mathrm{mg} / \mathrm{dL}$ & & $120 \mathrm{~min}$ & $156 \mathrm{mg} / \mathrm{dL}$ \\
\hline CK & $83 \mathrm{U} / \mathrm{L}$ & $\mathrm{HbA1c}$ & $7.9 \%$ & C-peptide & $0 \mathrm{~min}$ & $<0.2 \mathrm{ng} / \mathrm{mL}$ \\
\hline AST & $41 \mathrm{U} / \mathrm{L}$ & Glycoalbumin & $19.1 \%$ & & $120 \mathrm{~min}$ & $0.6 \mathrm{ng} / \mathrm{mL}$ \\
\hline ALT & $30 \mathrm{U} / \mathrm{L}$ & TSH & $1.13 \mu \mathrm{IU} / \mathrm{mL}$ & & & \\
\hline$\gamma$-GTP & $35 \mathrm{U} / \mathrm{L}$ & Free $\mathrm{T} 4$ & $1.23 \mathrm{ng} / \mathrm{dL}$ & & & \\
\hline ALP & 219 U/L & Free $\mathrm{T} 3$ & $2.6 \mathrm{pg} / \mathrm{mL}$ & & & \\
\hline
\end{tabular}

WBC: white blood cell, RBC: red blood cell, Hb: hemoglobin, PLT: platelet, TP: total protein, Alb: albumin, LDH: lactate dehydrogenase, CK: creatine kinase, AST: aspartate aminotransferase, ALT:alanine aminotransferase, $\gamma$-GTP: gamma glutamyl transpeptidase, ALP: alkaline phosphatase, T-Bil: total bilirubin, BUN: blood urea nitrogen, Cre:creatinine, eGFR: estimated glomerular filtration rate, UA: uric acid, CRP: C-reactive protein, TSH: thyroid stimulating hormone
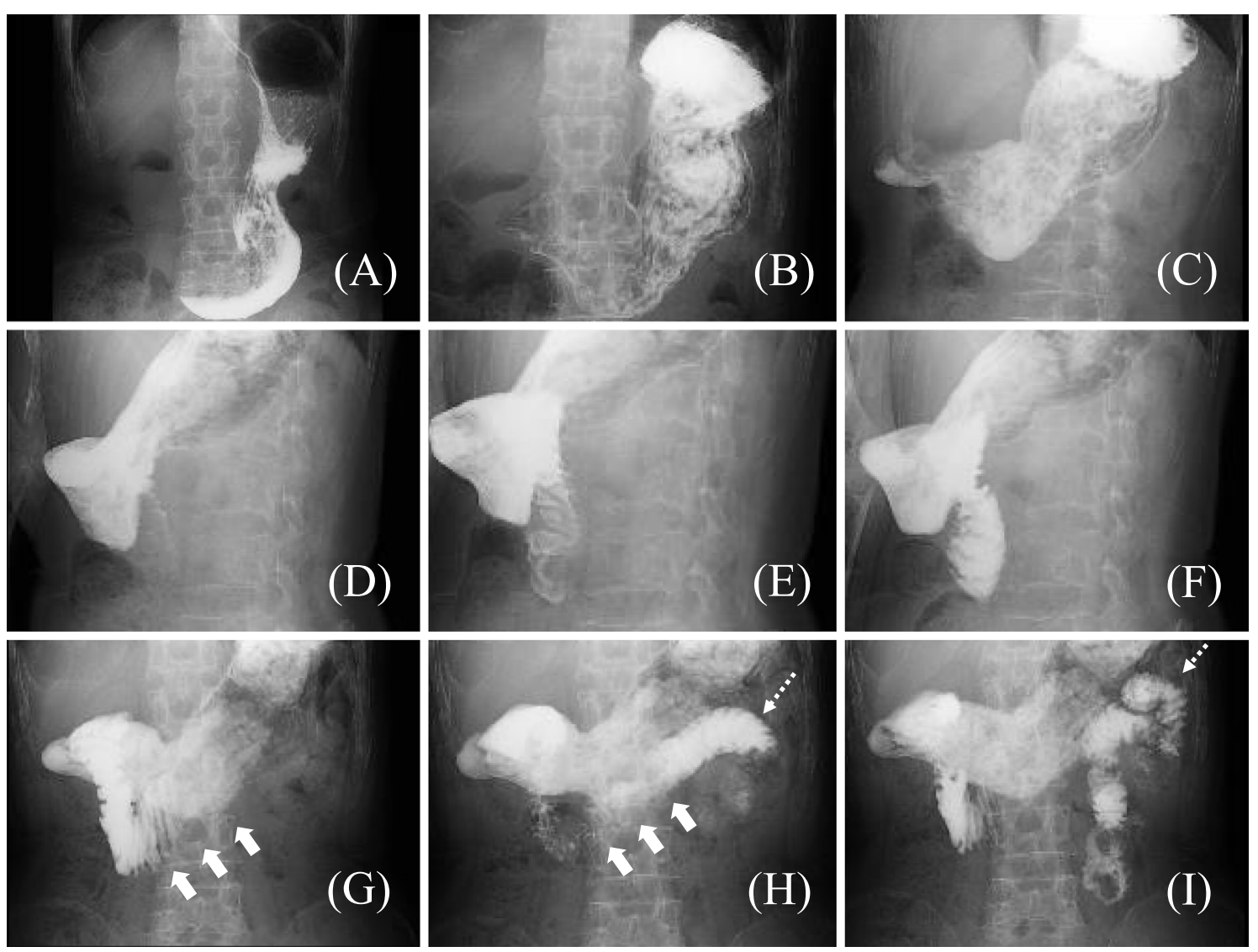

Figure 2. Gastric fluoroscopy findings obtained approximately every 30 seconds. A-B: The stomach is dilated despite fasting for more than $12 \mathrm{~h}$. C-F: Intestinal peristalsis from the stomach to duodenum is slightly decreased, and the contrast medium is also slow to pass. G-I: There is no obstruction at the horizontal part of the duodenum (arrowhead). Intestinal peristalsis distal to the duodenojejunal flexure (dotted arrowhead) is well-preserved. 
Clinical features

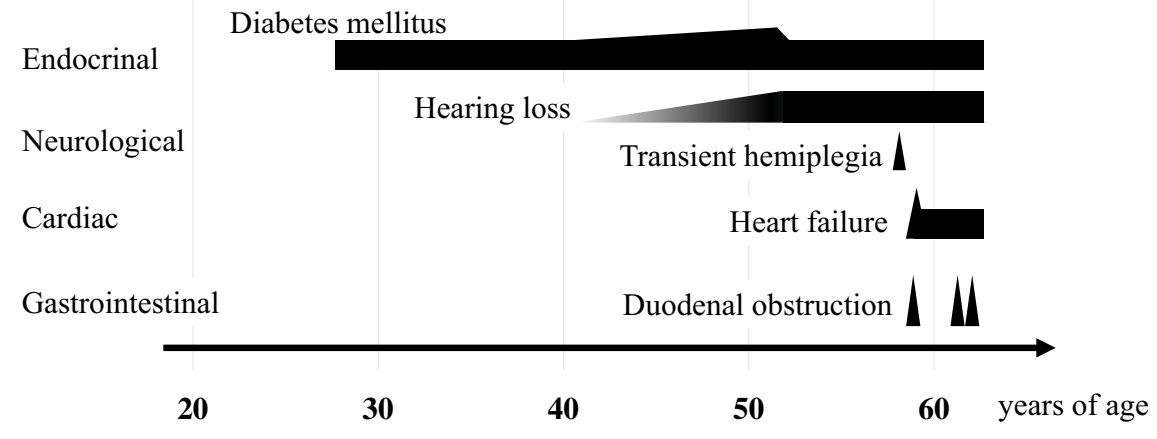

Figure 3. Clinical course of the patient.

\section{Discussion}

Gastrointestinal manifestations, such as a poor appetite, gastroesophageal sphincter dysfunction, constipation, dysphagia, vomiting, gastroparesis, diarrhea, pancreatitis, hepatopathy or gastrointestinal pseudo-obstruction, are common in mitochondrial diseases (13). Gastrointestinal dysfunction in our patient was transient and was confined to the horizontal part of the duodenum between the aorta and the superior mesenteric artery in all the episodes, and the condition was initially diagnosed as being caused by SMAS. SMAS is a rare medical condition with clinical symptoms resulting from vascular compression of the horizontal part of the duodenum in the angle between the aorta and the superior mesenteric artery. The complicated clinical signs of duodenal obstruction in this condition include postprandial epigastric pain, nausea, vomiting, anorexia, and weight loss. SMAS is more likely to be observed in young patients with rapid weight loss and certain metabolic alterations, along with decreased mesenteric and retroperitoneal fat mass, than in others. There are numerous predisposing conditions for SMAS, such as chronic wasting disease, eating disorders, postoperative state, and congenital anomalies (12).

CT angiography is useful for the diagnosis of SMAS. In a previous angiographic study of cases of SMAS, the aortomesenteric angle was $6^{\circ}-15^{\circ}$ (normal reported range, $25^{\circ}$ $60^{\circ}$ ), and the distance was $2-8 \mathrm{~mm}$ (normal reported range, $10-28 \mathrm{~mm}$ ) (14). Furthermore, interrupted flow of the contrast agent in the horizontal part of the duodenum by the superior mesenteric artery was observed on gastric fluoroscopy $(15,16)$. While CT angiography was not performed in the present case, and the aortomesenteric angle could not be precisely evaluated, shortening of the aortomesenteric distance was confirmed. However, gastric fluoroscopy did not show any duodenal obstruction due to compression by the superior mesenteric artery. A few cases of mitochondrial diseases with SMAS have been reported (17-19). In each of these reported cases, the patient had typical characteristics of SMAS, such as rapid weight loss or a young age. Although our patient was lean, he seemed to be an atypical candidate for SMAS, as he was much older in age than most previous patients and showed no recent weight loss.

CIPO is a syndrome characterized by recurrent episodes of clinical intestinal obstruction in the absence of obstructive lesions in the gastrointestinal tract (8). CIPO is caused by impairment of one or more factors regulating gastrointestinal motility, such as the myogenic, neurogenic, and/or mesenchymopathic system (20). Ohkubo et al. proposed the following diagnostic criteria: a) the onset of one or more symptoms of bowel obstruction at least 6 months prior to the diagnosis, b) abdominal bloating or abdominal pain or both for the previous 12 weeks, c) dilatation and/or air-fluid level of the intestine on abdominal X-ray, ultrasonography and/or computed tomography imaging, and d) no evidence of structural disease (20). In our present case, dilatation of the stomach and proximal duodenum without any mechanical obstructive causes were observed, accompanied by recurrent vomiting and duodenal obstruction for a few years. Thus, this case was considered to meet the diagnostic criteria of CIPO. CIPO occurs in all segments of the gastrointestinal tract from the esophagus to the rectum and mainly affects the small intestine (20). The segment of intestinal dysfunction can be localized depending on the types of diseases in CIPO, although previous studies have lacked data regarding the exact proportions of gut segments affected in CIPO. CIPO is thought to include patients who have been previously reported as "idiopathic megaduodenum". Among those patients with gastrointestinal obstruction localized to the duodenum, some of them had also been misdiagnosed with SMAS (21). Similarly, in the present case, CIPO was considered to involve the stomach and duodenum.

As mentioned above, CIPO has been classified into primary and secondary types. Secondary CIPO can occur in association with many multisystem disorders, including connective tissue diseases (systemic sclerosis), neurologic diseases (mitochondrial diseases, diabetic neuropathy, Parkinson's disease), drug-related conditions, paraneoplastic and postinfectious syndromes, and amyloidosis $(8,10)$.

A previous study reported that mitochondrial defects were identified in 15 patients of 80 CIPO patients, representing $19 \%$ of all cases (22). While secondary CIPO due to mitochondrial disease has been frequently observed, especially in patients with MELAS, CIPO might also be a significant 
complication in cases with well-controlled mitochondrial diseases, such as cardiomyopathy and encephalopathy (11). However, little is known about the pathological mechanism by which CIPO develops in cases of mitochondrial disease. Betts et al. reported profound cytochrome oxidase (COX) deficiency in the smooth muscle layers of all regions of the gastrointestinal tract in patients with the mitochondrial DNA $3243 \mathrm{~A}>\mathrm{G}$ mutation, although there was little evidence of morphologic abnormalities within the gastrointestinal tissues in these patients (23). Jeffrey et al. reported intestinal pseudo-obstruction in a patient with a mitochondrial defect in the $\beta$-oxidation cycle, hypothesizing that impairment of mitochondrial $\beta$-oxidation might play a role in the pathogenesis of intestinal pseudo-obstruction (24).

In our present case, the possibility of secondary CIPO derived from diabetic autonomic neuropathy or diabetic gastroparesis was also considered. Diabetic gastroparesis is mainly found in patients with long-standing diabetes mellitus, as a symptom of diabetic autonomic neuropathy, accompanied by a broad spectrum of symptoms and signs including nausea, bloating, loss of appetite, and postprandial vomiting (25). However, our patient had no significant signs of advanced diabetic neuropathy, including decreased $\mathrm{CV}_{\mathrm{R}-\mathrm{R}}$ as an indicator of diabetic autonomic neuropathy. Therefore, we considered that the CIPO in our case was secondary to mitochondrial diseases rather than being associated with diabetic neuropathy.

There is no specific treatment for CIPO, with the main treatments being nutritional support, pharmacologic treatment, and surgery $(26,27)$. Nutritional therapy includes small and frequent meals, easily digestible meals, and enteral nutrition using a feeding tube. The main aim of pharmacological treatment is to improve gastrointestinal motility, decrease symptom severity, and reduce bacterial overgrowth related to intestinal stasis. Many therapeutic agents are used in CIPO patients, such as serotoninergic agents, erythromycin, metoclopramide, and domperidone. Gastrostomy and jejunostomy can be effective in relieving gastrointestinal symptoms by decreasing the intestinal pressure. The effectiveness of bowel resection in CIPO patients is generally limited because the degree of bowel dysfunction might increase over time. If a patient is refractory or has difficulty continuing these treatments due to complications, small bowel transplantation may also be an option. While the lesion was localized to the duodenum at every onset in this patient, we decided to follow this patient with pharmacological treatment, as the risk of surgery was considered to be high due to complications associated with mitochondrial diseases.

In conclusion, we herein described a case of mitochondrial diseases with recurrent duodenal obstruction, who was initially diagnosed with SMAS but was later diagnosed with CIPO. If a patient with mitochondrial disease presents with recurrent gastrointestinal manifestations and imaging findings suggestive of SMAS, CIPO should be considered in the differential diagnosis.
The authors state that they have no Conflict of Interest (COI).

\section{References}

1. Schapira AH. Mitochondrial disease. Lancet 368: 70-82, 2006.

2. Taylor RW, Turnbull DM. Mitochondrial DNA mutations in human disease. Nat Rev Genet 6: 389-402, 2005.

3. Guillausseau PJ, Massin P, Dubois-LaForgue D, et al. Maternally inherited diabetes and deafness: a multicenter study. Ann Intern Med 134: 721-728, 2001.

4. El-Hattab AW, Adesina AM, Jones J, Scaglia F. MELAS syndrome: Clinical manifestations, pathogenesis, and treatment options. Mol Genet Metab 116: 4-12, 2015.

5. Goto Y, Nonaka I, Horai S. A mutation in the tRNA (Leu)(UUR) gene associated with the MELAS subgroup of mitochondrial encephalomyopathies. Nature 348: 651-653, 1990.

6. van den Ouweland JM, Lemkes HH, Ruitenbeek W, et al. Mutation in mitochondrial tRNA (Leu)(UUR) gene in a large pedigree with maternally transmitted type II diabetes mellitus and deafness. Nat Genet 1: 368-371, 1992.

7. Kadowaki T, Kadowaki H, Mori Y, et al. A subtype of diabetes mellitus associated with a mutation of mitochondrial DNA. N Engl J Med 330: 962-968, 1994.

8. Faulk DL, Anuras S, Christensen J. Chronic intestinal pseudoobstruction. Gastroenterology 74: 922-931, 1978.

9. Iida H, Ohkubo H, Inamori M, Nakajima A, Sato H. Epidemiology and clinical experience of chronic intestinal pseudoobstruction in Japan: a nationwide epidemiologic survey. J Epidemiol 23: 288-294, 2013.

10. Connor FL, Di Lorenzo C. Chronic intestinal pseudo-obstruction: assessment and management. Gastroenterology 130 (2 Suppl 1): S 29-36, 2006.

11. Sekino Y, Inamori M, Yamada E, et al. Characteristics of intestinal pseudo-obstruction in patients with mitochondrial diseases. World J Gastroenterol 18: 4557-4562, 2012.

12. Welsch T, Büchler MW, Kienle P. Recalling superior mesenteric artery syndrome. Dig Surg 24: 149-156, 2007.

13. Finsterer J, Frank M. Gastrointestinal manifestations of mitochondrial disorders: a systematic review. Therap Adv Gastroenterol 10: 142-154, 2017.

14. Gustafsson L, Falk A, Lukes PJ, Gamklou R. Diagnosis and treatment of superior mesenteric artery syndrome. Br J Surg 71: 499501, 1984.

15. Unal B, Aktaş A, Kemal G, et al. Superior mesenteric artery syndrome: CT and ultrasonography findings. Diagn Interv Radiol 11: 90-95, 2005.

16. Merrett ND, Wilson RB, Cosman P, Biankin AV. Superior mesenteric artery syndrome: diagnosis and treatment strategies. J Gastrointest Surg 13: 287-292, 2009.

17. Yee ML, Wong R, Datta M, et al. Mitochondrial disease: an uncommon but important cause of diabetes mellitus. Endocrinol Diabetes Metab Case Rep 2018, 2018.

18. Kishi T, Kawahara H, Tanaka $T$, et al. Superior mesenteric artery syndrome complicating mitochondrial encephalopathy. J Pediatr Gastroenterol Nutr 26: 464-467, 1998.

19. Kwon OY, Lim SG, Park SH. Mitochondrial encephalomyopathy, lactic acidosis, and stroke-like episode leading to recurrent superior mesenteric artery syndrome. Am J Emerg Med 32: 951.e951952, 2014.

20. Ohkubo H, Iida H, Takahashi H, et al. An epidemiologic survey of chronic intestinal pseudo-obstruction and evaluation of the newly proposed diagnostic criteria. Digestion 86: 12-19, 2012.

21. Schuffler MD. Chronic Intestinal Pseudo-Obstruction Syndromes. Medical Clinics of North America 65: 1331-1358, 1981.

22. Amiot A, Tchikviladzé M, Joly F, et al. Frequency of mitochondrial defects in patients with chronic intestinal pseudo-obstruction. 
Gastroenterology 137: 101-109, 2009.

23. Betts J, Barron MJ, Needham SJ, Schaefer AM, Taylor RW, Turnbull DM. Gastrointestinal tract involvement associated with the $3243 \mathrm{~A}>\mathrm{G}$ mitochondrial DNA mutation. Neurology 70: 12901292, 2008.

24. Gilbert J, Ibdah JA. Intestinal pseudo-obstruction as a manifestation of impaired mitochondrial fatty acid oxidation. Med Hypotheses 64: 586-589, 2005.

25. Pop-Busui R, Boulton AJ, Feldman EL, et al. Diabetic Neuropathy: A Position Statement by the American Diabetes Association. Diabetes Care 40: 136-154, 2017.

26. De Giorgio R, Cogliandro RF, Barbara G, Corinaldesi R,
Stanghellini V. Chronic Intestinal Pseudo-Obstruction: Clinical Features, Diagnosis, and Therapy. Gastroenterol Clin North Am 40: 787-807, 2011

27. Di Nardo G, Di Lorenzo C, Lauro A, et al. Chronic intestinal pseudo-obstruction in children and adults: diagnosis and therapeutic options. Neurogastroenterol Motil 29: 1-13, 2017.

The Internal Medicine is an Open Access journal distributed under the Creative Commons Attribution-NonCommercial-NoDerivatives 4.0 International License. To view the details of this license, please visit (https://creativecommons.org/licenses/ by-nc-nd/4.0/).

(C) The Japanese Society of Internal Medicine Intern Med Advance Publication 